\title{
Serum micro-rna profiles in patients with autosomal dominant polycystic kidney disease according to hypertension and renal function
}

\author{
Ismail Kocyigit ${ }^{1}$, Serpil Taheri², Elif Funda Sener ${ }^{2}$, Eray Eroglu', Fahir Ozturk ${ }^{3}$, Aydin Unal', Kezban Korkmaz ${ }^{4}$, \\ Gokmen Zararsiz', Murat Hayri Sipahioglu', Yusuf Ozkul ${ }^{4}$, Bulent Tokgoz ${ }^{1}$, Oktay Oymak' $^{1}$, Tevfik Ecder ${ }^{6}$ \\ and Jonas Axelsson $7,8^{*}$ (D)
}

\begin{abstract}
Background: Autosomal dominant polycystic kidney disease (ADPKD) is a common hereditary disorder with unclear disease mechanism. Currently, overt hypertension and increased renal volume are the best predictors of renal function. In this study, we assessed the usefulness of selected circulating microRNAs (miRs) to predict disease progress in a cohort with ADPKD.

Methods: Eighty ADPKD patients ( $44.6 \pm 12.7$ years, 40\% female, $65 \%$ hypertensive) and 50 healthy subjects (HS; $45.4 \pm 12.7,44 \%$ female) were enrolled in the study. Serum levels of 384 miRs were determined by Biomark Real Time PCR. Groups were compared using the limma method with multiple-testing correction as proposed by Smyth (corrected $p<0.01$ considered significant).

Results: Comparing ADPKD to HS, we found significant differences in blood levels of 18 miRs (3 more and 15 less abundant). Of these, miR-3907, miR-92a-3p, miR-25-3p and miR-21-5p all rose while miR-1587 and miR-3911 decreased as renal function declined in both cross-sectional and longitudinal analysis. Using ROC analysis, an increased baseline miR-3907 in the circulation predicted a $>10 \%$ loss of GFR over the following 12 months (cut-off $>2.2 \mathrm{AU}$, sensitivity $83 \%$, specificity $78 \%$, area 0.872 [95\% Cl: 0.790-0.953, $p<0.001$ ]). Adjusting for age and starting CKD stage using multiple binary logistic regression analysis did not abrogate the predictive value.
\end{abstract}

Conclusion: Increased copy numbers of miR-3907 in the circulation may predict ADPKD progression and suggest pathophysiological pathways worthy of further study.

\section{Background}

Autosomal dominant polycystic kidney disease (ADPKD) is a congenital progressive disease resulting in chronic kidney disease (CKD) that often progresses to end-stage renal disease (ESRD). In most patients, renal function is sufficient to live a normal life until the fourth to sixth decades of life but thereafter declines [1]. The main

\footnotetext{
* Correspondence: jonas.axelsson@ki.se

${ }^{7}$ Division of Vascular Biology, Department of Medical Biochemistry and Biophysics, Karolinska institutet, Karolinska University Hospital, Stockholm, Sweden

${ }^{8}$ Department of Clinical Immunology, Karolinska University Hospital, C2:66 ImmTrans, 14186 Stockholm, Sweden

Full list of author information is available at the end of the article
}

predictors of future renal function are the presence of hypertension, increasing kidney volume and decreased renal blood flow [2]. Of these hypertension often significantly predates the development of chronic kidney disease (CKD) [1], while imaging studies to detect the size and amount of remaining parenchyma of the kidneys are resource consuming and not very sensitive $[1,3]$. Identifying patients at risk has obvious clinical application and the identification of relevant biomarkers associated with ADPKD CKD-development may also help to elucidate the currently obscure mechanisms linking commonly encountered mutations in the PKD1 and 2 genes to renal cysts and CKD [1]. 
MicroRNAs (miRs) are small, non-coding RNA molecules that modulate many different intracellular pathways negatively regulating gene expression at the posttranscriptional level. As each miR has multiple putative targets, and as the distribution of miRs vary between cell types and over time, they are likely to play a physiological role [4]. In renal tissue miRs have been implicated in both embryonic development and kidney disease processes and altered levels have been reported in the blood and urine during several specific glomerular and tubular diseases as well as in animal models of these [5-8]. While few studies have investigated miRs in ADPKD, two recent animal experiments have suggested that they may be important as regulators of pro-fibrotic calcium signaling $[9,10]$.

In the present pilot study, we asked if circulating miRs would differentiate ADPKD patients from healthy subjects under clinically relevant conditions, and if levels of any circulating miR would predict the furure clinical course of ADPKD during one year of follow-up.

\section{Methods}

\section{Study design and patient selection}

Among the patients with ADPKD included in the Turkish Society of Nephrology's Polycystic Kidney Disease Working Group Registry, we screened those scheduled to receive followed-up by the Medical Faculty of Erciyes University between June 2013 and January 2015. The study was approved by the Erciyes University Ethical Review Board (Kayseri, Turkey) prior to start, and all participants signed written informed consent forms after being informed about the study and given time to consider their participation.

Eligible patients were those aged 18-70 years under regular follow-up and meeting the above diagnostic criteria for ADPKD. Patients with a known glomerular filtration rate (eGFR) $<15 \mathrm{ml} / \mathrm{min} / 1.73 \mathrm{~m}^{2}$ and those with diagnosed cardiovascular disorders, diabetes mellitus or an active infection were all excluded. The patients were invited to enroll by post, and those that accepted all signed written informed consent forms. Demographic characteristics (e.g. sex, age, education and smoking history), renal manifestations (e.g. hematuria, urinary system infection, urinary tract stones and renal replacement therapy) and cardiovascular manifestations (e.g. hypertension and mitral valve prolapse) were hen recorded using a web-based data collection form. Using the above criteria, 80 ADPKD patients were included in the study. A total of 50 healthy subjects were also recruited by advertisement and gave written, informed consent to participate. Both patients and controls were asked to undergo ambulatory blood pressure monitoring to diagnose the presence or absence of hypertension. Data of $24 \mathrm{~h}$ urine samples was collected at inclusion and in the patients again after exactly 12 months of follow-up $(n=80)$ to determine eGFR (mean of urinary creatinine and urea clearances) and proteinuria levels.

\section{Continuous blood-pressure monitoring}

Twenty-four-hour blood pressure monitoring was performed using a Del Mar Medical Ressurometer Model P6 (Del Mar Reynolds, Irvine, CA, USA), and the results were assessed using the companion software. Ambulatory measurements were conducted once every $15 \mathrm{~min}$ from 7 a.m. until 11 p.m., and once every $30 \mathrm{~min}$ from 11 p.m. until 7 a.m. Evaluation was performed taking the mean values of day and night blood pressures into account. Hypertension was considered to be present if the average systolic pressure was $\geq 130 \mathrm{mmHg}$ and/or the average diastolic pressure was $\geq 80 \mathrm{mmHg}$ over the $24-\mathrm{h}$, or if the individual was taking antihypertensive medication $(n=10)$.

\section{RNA isolation}

Five milliliters of venous blood was drawn and after coagulation centrifuged at $4000 \mathrm{~g}$ for $15 \mathrm{~min}$ to isolate serum, which was transferred into clean microcentrifuge tubes and again centrifuged (12,000 g, $5 \mathrm{~min}$ ) with the resulting serum aliquoted into $200 \mu \mathrm{l}$ and stored at $-80^{\circ} \mathrm{C}$ until RNA isolation. Total RNA was isolated using Trizol $(500 \mu \mathrm{L})$ (Roche, Mannheim, Germany) according to the manufacturer's instructions and stored at $-80{ }^{\circ} \mathrm{C}$.

\section{Reverse transcription (RT) and quantitative PCR}

Isolated RNA samples were reverse-transcribed into complementary DNA (cDNA) in $5 \mu$ l final reaction volumes using MicroRNA Reverse Transcription Kit (miScript II RT Kit, Cat No: 218,161, Qiagen, Germany). cDNA samples were kept at $-80{ }^{\circ} \mathrm{C}$ until PCR analysis. Next, we performed pre-amplification using the PreAmp Master Mix (miScript Microfluidics PreAMP Kit, Cat No: 331,455, Qiagen) together with the miScript miRNA PCR Array Human Serum \& Plasma 384HC (Cat.No: MIHS-3106Z, Qiagen, Germany) (Additional file 1). For pre-amplification $2 \mu \mathrm{l} 1 / 5$ diluted RT product were added to $3 \mu \mathrm{l}$ of the PreAmp mix. PreAmp Thermal protocol were as follows: $95{ }^{\circ} \mathrm{C}$ for $15 \mathrm{~min}$, followed $94{ }^{\circ} \mathrm{C}$ for $120,55^{\circ} \mathrm{C}$ for $60 \mathrm{~s}$ and $70{ }^{\circ} \mathrm{C}$ for 60 s by 2 cycles, followed by 10 cycles with $94{ }^{\circ} \mathrm{C}$ for $30 \mathrm{~s}, 60^{\circ} \mathrm{C}$ for $3 \mathrm{~min}$, finally $99.9^{\circ} \mathrm{C}$ for $600 \mathrm{~s}$, and a rest period at $4{ }^{\circ} \mathrm{C}$.

Next, qPCR was performed using the high-throughput BioMark Real-Time PCR system (Fluidigm, South San Francisco, CA, USA). Preamplified cDNA samples were diluted with low EDTA (0.1 mM) TE Buffer (1:5). A BioMark IFC controller HX (Fluidigm, South San Francisco, CA, USA) was used to mix a total of $3.15 \mu$ l diluted preAmplified cDNA with appropriate amounts of Universal PCR Master Mix, (miScript Microfluidics PCR Kit, Cat No: 331,431, Qiagen) and 20X GE Sample Loading 
Table 1 Comparison of demographical and biochemical data between ADPKD patients $(n=80)$ and healthy controls $(n=50)$

\begin{tabular}{|c|c|c|c|}
\hline Clinical Parameters & ADPKD patients $(n=80)$ & Healthy controls $(n=50)$ & $p$ \\
\hline Age, year & $44.6 \pm 12.7$ & $45.4 \pm 12.7$ & 0.35 \\
\hline Gender, F/M & $33 / 47$ & $22 / 28$ & 0.12 \\
\hline Hemoglobin, g/L & $13.9 \pm 2.1$ & $13.3 \pm 1.9$ & 0.74 \\
\hline BMI $\left(\mathrm{kg} / \mathrm{m}^{2}\right)$ & $27.6 \pm 5.37$ & $26.74 \pm 7.8$ & 0.21 \\
\hline Average 24-h systolic BP, mmHg & $139 \pm 7.2$ & $119 \pm 6.1$ & 0.005 \\
\hline Average 24-h diastolic BP, mmHg & $86 \pm 4.8$ & $73 \pm 3.9$ & 0.01 \\
\hline eGFRa ${ }^{a}, \mathrm{~mL} / \mathrm{min}$ per $1.73 \mathrm{~m}^{2}$ & $74.0 \pm 35.4$ & $98.1 \pm 15.3$ & $<0.001$ \\
\hline Smoking, $n(\%)$ & $18(22)$ & $10(20)$ & 0.54 \\
\hline Fasting Total cholesterol, mg/dL & $197 \pm 39$ & $184 \pm 33$ & 0.19 \\
\hline HDL- cholesterol, mg/dL & $42 \pm 10$ & $46 \pm 8$ & 0.23 \\
\hline LDL- cholesterol, mg/dL & $124 \pm 30$ & $119 \pm 36$ & 0.63 \\
\hline Proteinuria, g/day & $0.47(0.12-1.19)$ & $0.15(0.07-0.6)$ & $<0.001$ \\
\hline $\mathrm{Hs}-\mathrm{CRP}, \mathrm{mg} / \mathrm{L}$ & $6.9 \pm 2.7$ & $3.5 \pm 1.6$ & 0.01 \\
\hline Albumin (g/dL) & $3.9 \pm 0.61$ & $4.1 \pm 0.62$ & 0.72 \\
\hline
\end{tabular}

ADPKD Autosomal-dominant polycystic kidney disease, eGFR estimated glomerular filtration rate, F/M Female/Male, HDL High density lipoprotein, Hs-CRP High sensitive C-reactive protein, $L D L$ Low density lipoprotein

${ }^{a}$ Calculated by the CKD-EPI formula

Data are expressed as mean \pm SD or median for normally distributed data and percentage (\%) for categorical variables.

Bold values indicate the significant values $(p<0.05)$

Reagent (Fluidigm, South San Francisco, CA, USA) in a Dynamic 96.96 array plate microfluidic system (Fluidigm,South San Francisco, CA, USA). Real-Time PCR was performed automatically using a thermal mixing protocol followed by $50{ }^{\circ} \mathrm{C}$ for $120 \mathrm{~s}, 70{ }^{\circ} \mathrm{C}$ for $1800 \mathrm{~s}$, $25{ }^{\circ} \mathrm{C}$ for $600 \mathrm{~s}$, then followed $95{ }^{\circ} \mathrm{C}$ for $600 \mathrm{~s}$. Finally, 23 cycles with $94{ }^{\circ} \mathrm{C}$ for $15 \mathrm{~s}, 55{ }^{\circ} \mathrm{C}$ for $30 \mathrm{~s}, 70{ }^{\circ} \mathrm{C}$ for $30 \mathrm{~s}$ followed by $60{ }^{\circ} \mathrm{C}$ for $60 \mathrm{~s}$. All of the genetic studies were performed by experienced operators in the Genome and Stem Cell Center at Erciyes University (GENKOK).

\section{Data collection and statistical analyses}

Data were collected with the Fluidigm ${ }^{\circ}$ Real-Time PCR analysis software using the linear baseline correction method and the auto global Cq threshold. System given

Table 2 Comparison of demographical and laboratory features between the 28 ADPKD patients without (HT-) and the 52 patients with (HT+) hypertension (defined as average systolic pressure was $\geq 130 \mathrm{mmHg}$ and/or the average diastolic pressure was $\geq 80 \mathrm{mmHg}$ during $24-\mathrm{h}$, or if the individual was taking antihypertensive medication $[n=10]$ )

\begin{tabular}{|c|c|c|c|}
\hline \multirow[t]{2}{*}{ Variables } & \multicolumn{2}{|l|}{ ADPKD patients $(n=80)$} & \multirow[t]{2}{*}{$p$} \\
\hline & Non-hypertensives $(n=28)$ & Hypertensives $(n=52)$ & \\
\hline Age (years) & $32.7 \pm 8.8$ & $47.9 \pm 11.4$ & $<0.001$ \\
\hline Gender (male) & $15(53.0)$ & $32(61)$ & 0.07 \\
\hline Hemoglobin, g/l & $13.4 \pm 1.5$ & $14.0 \pm 1.7$ & 0.22 \\
\hline BMI $\left(\mathrm{kg} / \mathrm{m}^{2}\right)$ & $26.6 \pm 5.37$ & $29.74 \pm 7.8$ & 0.109 \\
\hline Average 24-h systolic BP, mmHg & $124 \pm 4.6$ & $150 \pm 9.1$ & $<0.001$ \\
\hline Average $24-\mathrm{h}$ diastolic BP, $\mathrm{mmHg}$ & $73 \pm 3.9$ & $98 \pm 7.4$ & $<0.001$ \\
\hline Smoking status, $n(\%)$ & $6(21)$ & $12(23)$ & 0.701 \\
\hline eGFR $\mathrm{ml} / \mathrm{min} / 1.73 \mathrm{~m}^{2}$ & $108(85-116)$ & $58.5(21-91)$ & $<0.001$ \\
\hline Fasting total cholesterol, mg/dl & $178.7 \pm 41.7$ & $201.79 \pm 33.00$ & 0.02 \\
\hline Fasting LDL-C, mg/dl & $113.3 \pm 40.4$ & $124.3 \pm 30.6$ & 0.17 \\
\hline Proteinuria, g/day & $0.20(0.12-0.33)$ & $0.70(0.4-1.19)$ & $<0.001$ \\
\hline Albumin (g/dL) & $4.0 \pm 0.61$ & $3.9 \pm 0.54$ & 0.66 \\
\hline
\end{tabular}

ADPKD Autosomal-dominant polycystic kidney disease, BMI Body mass index, eGFR estimated glomerular filtration rate, $L D L$ Low density lipoprotein

Values are expressed as $n(\%)$, mean $\pm \mathrm{SD}$ or median(1st-3rd quartiles).

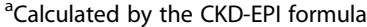

Bold values indicate the significant values $(p<0.05)$ 
Cq values of 999 and values larger than 23 haploid genome equivalents (HGEs) were considered as unreliable and removed. Median limit of detection (LOD) $\mathrm{Cq}$ values were calculated across all arrays to impute missing values. Data normalization was performed by using the $2^{-\Delta \Delta C T}$ method. To detect the differentially expressed miRs, the linear models for microarray and RNA-Seq data (limma) procedure was used along with false discovery rate correction of $p$ values according to Smyth [11]. Adjusted $p$ values less than 0.01 were considered statistically significant.

The DIANA-mirPath software v. 3.0 was used to identify signaling pathways associated with differentially expressed miRs [12] with highly conserved and experimentally verified TarBase v. 7.0 miRs were considered as potential targets and the agglomerative hierarchical clustering algorithm was applied to determine miR and pathway clusters based on their interaction levels.
Analyses were conducted using the Rcmdr and limma packages of R v. 3.1.2 (R Core Team, 2014).

To assess the performance metrics of miRs associated with changes in eGFR, ROC curve analysis was conducted. Moreover, univariate and multiple binary logistic regression models were constructed. Age, gender, hypertension, CKD and miR-3907 were included forced into an initial model which was used to identified the best independent subset using forward elimination with maximum likelihood ratio. Odds ratios are given with 95\% confidence intervals for each factor in each model.

\section{Results}

\section{Cohort demographics}

Age and gender distributions were similar in the control and patient groups (Table 1). The mean patient eGFR of $74.0 \pm 35.4 \mathrm{ml} / \mathrm{min} / 1.73 \mathrm{~m}^{2}$ was significantly lower than the $98.1 \pm 15.3 \mathrm{ml} / \mathrm{min} / 1.73 \mathrm{~m}^{2}$ mean value in control
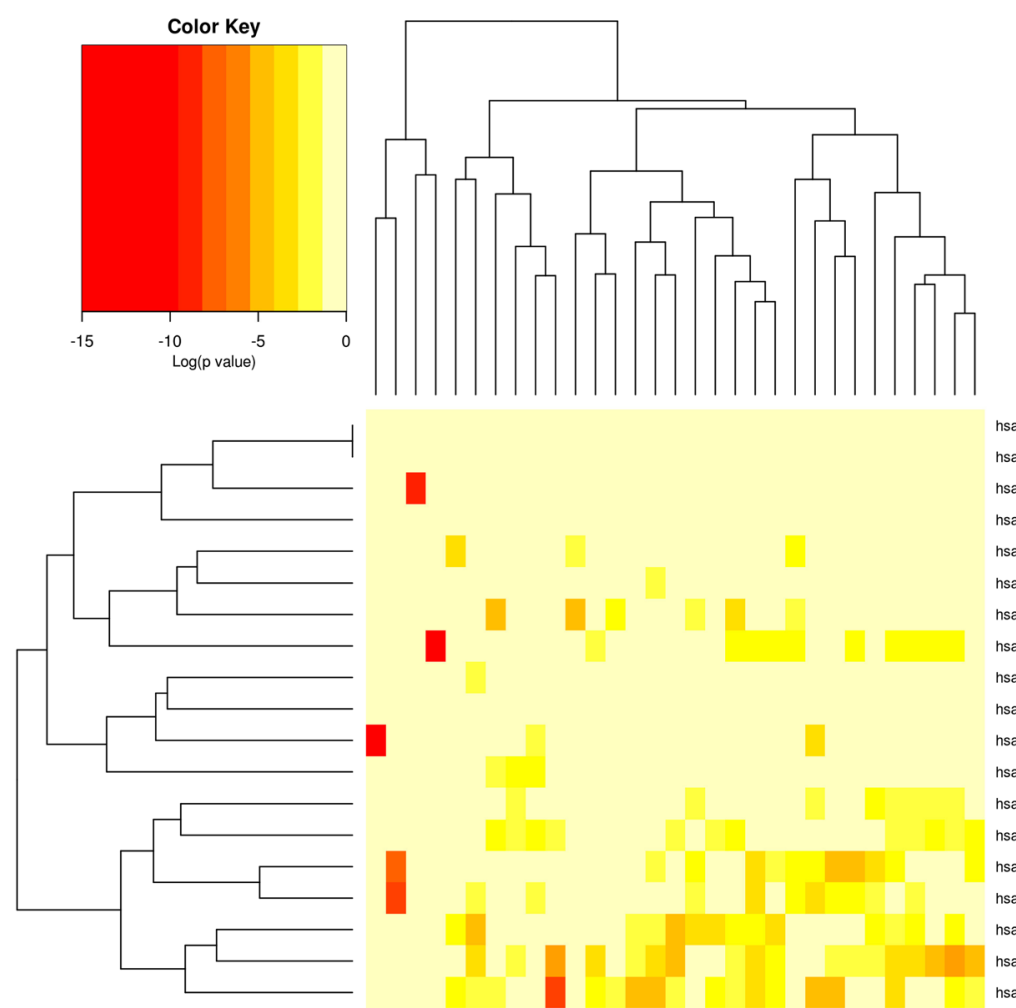

nsa-miR-4732-5p/microT-CDS nsa-miR-4732-5p/microT-CDS hsa-miR-3689a-5p/microT-CDS hsa-miR-4296/microT-CDS nsa-miR-1260a/microT-CDS hsa-miR-18a-5p/microT-CDS hsa-miR-145-5p/microT-CDS nsa-miR-4516/microT-CDS nsa-miR-1587/microT-CDS hsa-miR-4286/microT-CDS hsa-miR-629-3p/microT-CDS nsa-miR-3907/microT-CDS hsa-miR-365a-3p/microT-CDS hsa-miR-224-5p/microt-CDS nsa-miR-92a-3p/microT-CDS hsa-miR-25-3p/microT-CDS hsa-miR-3911/microT-CDS hsa-miR-126-5p/microt-CDS nsa-miR-27a-3p/microT-CDS

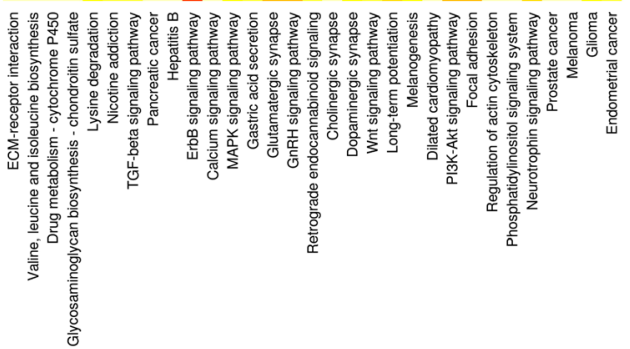

Fig. 1 Heatmap-plot of FDR-corrected log(p-values) for differences in venous blood miRs between 80 ADPKD patients and 50 healthy controls grouped by gene ontology group (GO). Generated using DIANA-miRPath v. 3.0 (40) 


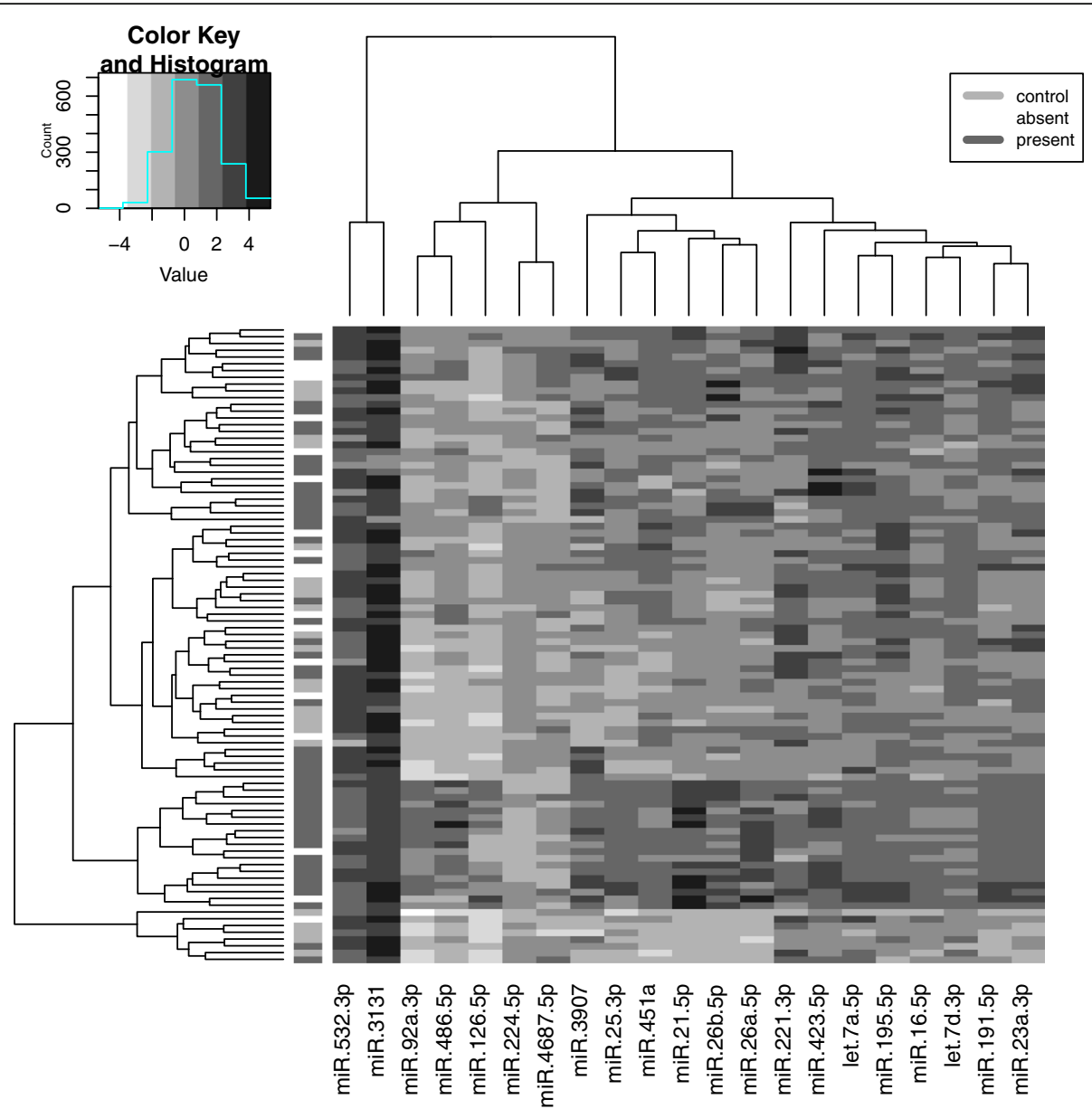

Fig. 2 Heatmap-plot displaying clustering of miRNA levels across study participants expressed as fold-change between all patients and all controls. Along the top is a dendrogram showing similarities between miRNAs, while the dendrogram along the left side shows similarities between groups (28 non-hypertensive and 52 hypertensive ADPKD patients and 50 non-hypertensive healthy controls)

subjects $(p<0.05)$. According to the CKD classification, 30 ADPKD were stage 1 (GFR $\geq 90 \mathrm{~mL} / \mathrm{min} / 1.73 \mathrm{~m}^{2}$ but albuminuria or hematuria), 22 in stage $2(60-89 \mathrm{~mL} /$ $\left.\mathrm{min} / 1.73 \mathrm{~m}^{2}\right), 20$ in stage $3\left(30-59 \mathrm{~mL} / \mathrm{min} / 1.73 \mathrm{~m}^{2}\right)$, and 8 in stage $4\left(15-29 \mathrm{~mL} / \mathrm{min} / 1.73 \mathrm{~m}^{2}\right)$. None of the healthy subjects had proteinuria or CKD.

Comparing hypertensive $(\mathrm{HT}+)$ to non-hypertensive (HT-) ADPKD patients only (Table 2; based on 24-h blood pressure data), HT+ were older $(47.9 \pm 11.4$ vs. $32.7 \pm 8.8$ years $)$ and had a lower GFR (58.5 [21-91] vs. $108[85-116] \mathrm{mL} / \mathrm{min} / 1.73 \mathrm{~m}^{2}$ ) than did HT-. Baseline proteinuria did not differ between these groups, nor did the mean proteinuria of the whole patient cohort change longitudinally during follow-up (baseline 0.47 (0.12$1.19)$ vs $0.54(0.15-1.32) \mathrm{g} / \mathrm{L}$ at 1 year $)$.

\section{miR levels in the venous blood of patients and healthy controls}

Comparing measured whole blood miR levels copy numbersof 18 miRs differed (corrected $p<0.01$ considered significant; -1.1 to 3.3 fold difference; Figs. 1 and 2). Of these, miR-25-3p, miR-92a-3p and miR-3907 were more abundant while miR-18a-5p, miR-27a-3p, miR-128-3p, miR-145-5p, miR-202-3p, miR-224-5p, miR-365a-3p, miR-629-3p, miR-1587, miR-3689-5p, miR-3911, miR4286, miR-4296, miR-4516 and miR-4732-5p were all less abundant (Fig. 2; $p<0.01$ for all).

\section{miR levels in ADPKD patients with our without hypertension}

Based on 24-h continous blood-pressure monitoring, patients were grouped into hypertensive $(\mathrm{HT}+$; average $\mathrm{SBP} \geq 130 \mathrm{mmHg}$ and/or average $\mathrm{DBP} \geq 80 \mathrm{mmHg}$ or taking antihypertensive medication $[n=10]$ ) or nonhypertensive (HT-). Comparing miRNAs copy numbers between these groups, patients with hypertension had signficantly different levels of 21 miRs when compared to non-hypertensives (Fig. 3A; -1.5 to 2.8 fold difference). Of these, miR-21-5p, miR-25-3p, miR- 26a-5p, miR-26b-5p, miR-92a-3p, miR-126-5p, miR-191-5p, 


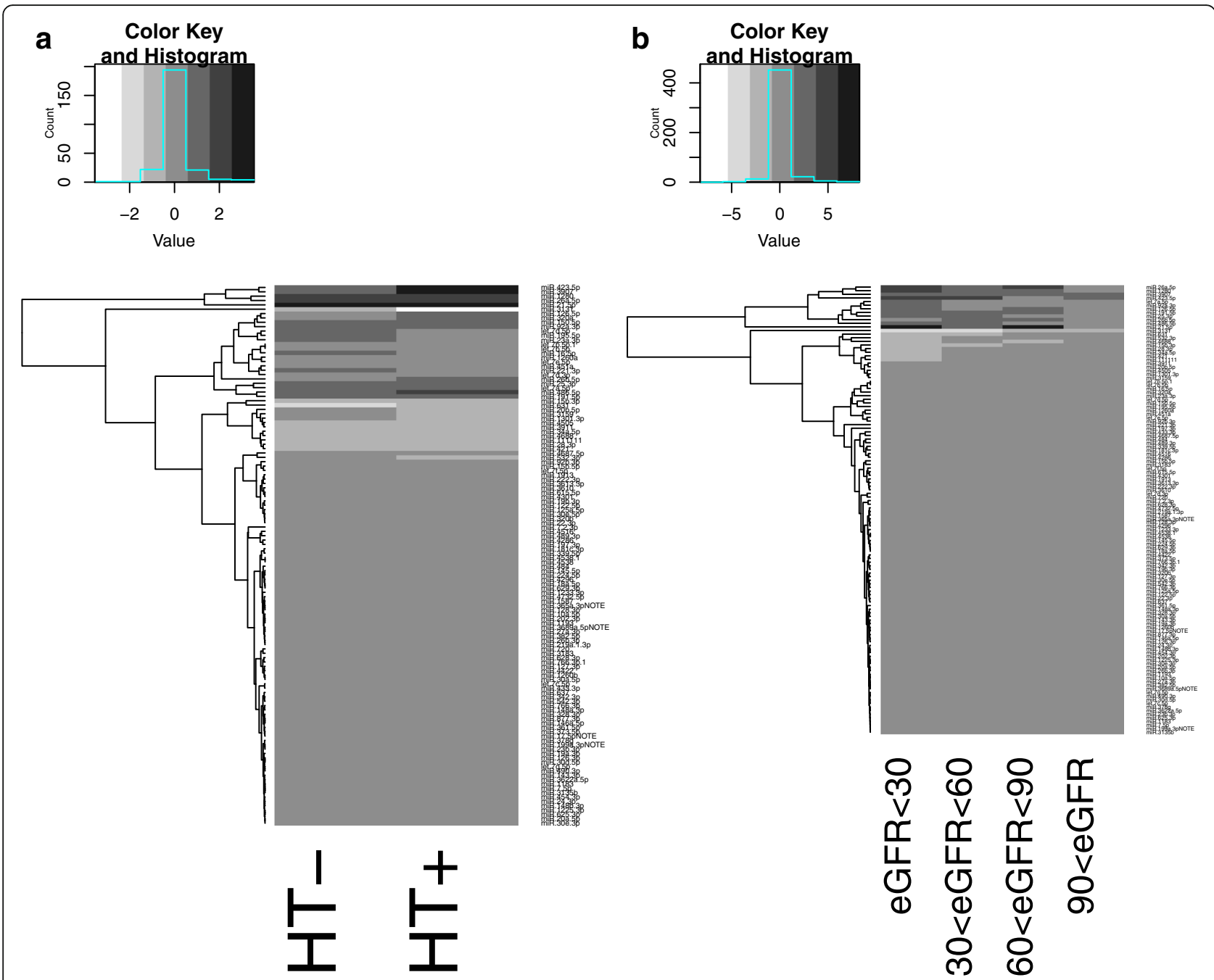

Fig. 3 Comparisons of measured differences in whole blood miR levels between a ADPKD patients with (HT+; $n=52)$ or without (HT-; $n=28)$ hypertension, and $\mathbf{b}$ ADPKD patients with CKD stage 1, 2, 3 or 4

miR-224-5p, miR-423-5p, miR-486-5p, miR-532-3p, miR-3907 and miR4687-5p were more abundant while let-7a-5p, let-7d-3p, miR-16-5p, miR-23a-3p, miR-1955p, miR-221-3p, miR-451a, miR-3131 were less abundant (corrected $p<0.01$ for all). However, these differences were not obvious when $\mathrm{HT}+$ and $\mathrm{HT}$ - patients were compared with controls (Fig. 3a).

\section{miR levels in ADPKD patients according to CKD stage}

When grouped according to CKD stage, we found 8 miRs that showed a dose-response behavior with incremental changes at least twice as GFR declined. These were miR1260a, miR-1587, miR-21-5p, miR-3907, miR-3911 and miR-92a-3p (Figs. 3b, 4). Of these, there was a consitent negative linear relationship between eGFR and blood copy numbers for miR-92a-3b and miR-3907 and a positive linear relationship between eGFR and blood copy numbers for miR-3911 and miR-1587. The remaining miRNAs showed a non-linear pattern.

\section{Patients' renal function after 1 year and the predictive value of baseline blood miRNAs}

As expected, there was a small but statistically significant decrease in eGFR $(74 \pm 35$ to $69 \pm 32 \mathrm{~mL} / \mathrm{min} /$ $1.73 \mathrm{~m}^{2} ; p<0.001$ ) during follow-up. Using data of baseline and follow-up eGFR, we estimated each patients' decline during the year (median -5 [range +2 to -29 ] $\mathrm{mL} / \mathrm{min} / 1.73 \mathrm{~m}^{2} /$ year). Patients were then grouped into two groups according to if their loss over the period was $\leq 10 \%$ or $>10 \%$ of their baseline value. 30 patients had progression rate above $10 \%$.

Next, we assessed the relative predictive value of the miRs that differed significantly between hypertensive and non-hypertensive ADPKD to predict the future loss of eGFR $>10 \%$. Of these, only miR-3907 was significantly 


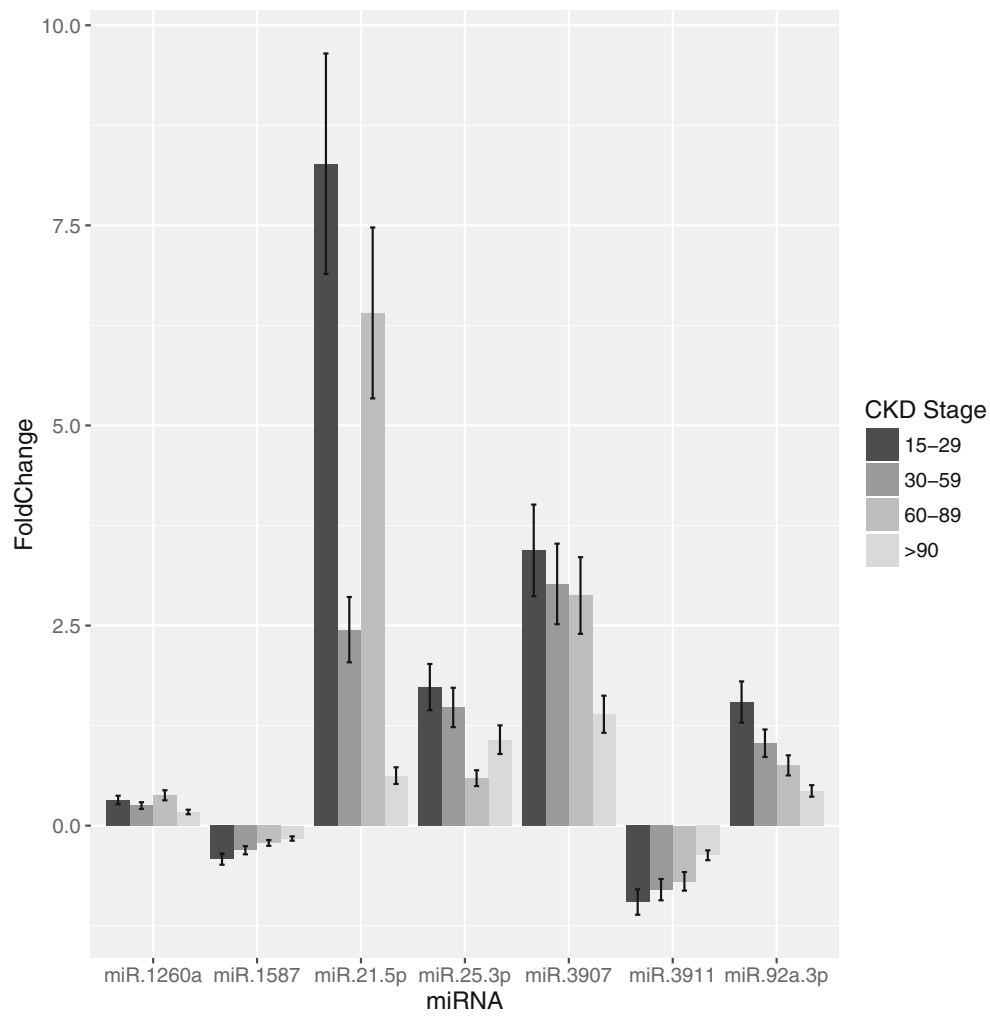

Fig. 4 Comparison of circulating levels of selected miRs between patients with various stages of CKD. Adjusted $p$-values computed based on Benjamini-Hochberg correction are reported

predictive in ROC analysis (Fig. 5). The sensitivity and specificity of miR-3907 were $83 \%$ and $78 \%$ (cut-off $>2.2 \mathrm{AU})$ with an area of under the ROC curve of 0.872 (95\% CI: 0.790-0.953, $p<.0001$ ).

Additionally, we performed univariate and multiple binary logistic regression analysis comparing the realtionship between selected variables and progression outcomes in all patients. A loss of eGFR $>10 \%$ during follow-up was independently associated with age, CKD stage and miR-3907 (Table 3).

\section{Discussion}

The present study was undertaken in order to investigate the potential use of circulating miRs as biomarkers of the future clinical course of ADPKD patients. Autosomal dominant polycystic kidney disease (ADPKD) is the most common genetic cause of chronic kidney disease $[1,13]$. While the disease mechanisms remain unclear, both the causal mutation, renal volume and the amount of hypertension are considered surrogate markers of progression while controlling hypertension is today the only widely accepted treatment $[1,13]$.

We found significant differing levels of multiple miRs both between ADPKD patients and healthy controls, as well as between ADPKD hypertensives ( $\mathrm{HT}+$ ) and nonhypertensives (HT-). In each case the differences were at most 3-fold, while the overlap between $\mathrm{HT}+$ and HTgroups was significant and apparently not associated with blood pressure (Fig. 3a). Differences between CKD stages tended to be higher (up to 7-fold; Fig. 3b) and more systematic (Figs. 3, 4). Thus, our data suggests that as with most other biomarkers, renal function is an important consideration when using circulating miRs as biomarkers. It is nonetheless encouraging that at least one miR (miR-3907) showed a robust association with progression of eGFR decline in the patient group (Fig. 5). Copy numbers of miR-3907 were 2.7-fold higher in ADPKD patients than in healthy controls, with a 1.2-fold increase in ADPKD patients with hypertension as compared to ADPKD patients without hypertension. In addition, ADPKD patients with high copy numbers of miR-3907 were also more likely to lose $>10 \%$ of their residual GFR during the one year follow-up.

While miR-3907 is not predicted to target mRNA derived from PKD1 or 2, the canonical ADPKD-associated genes, it is highly likely to bind to mRNA of the inositol 1,4,5-trisphosphate receptor (IP3R) interacting protein (IP(3)RIP) [14]. This is of interest as the receptor target of IP(3)RIP, called IP(3)R, is an intracellular $\mathrm{Ca}^{2+}$-channel in the endoplasmic reticulum reported to be a main effector of PKD 1 and 2-induced calcium signaling [15]. Indeed, the PKD 2-IP(3)R interaction may be instrumental for the 


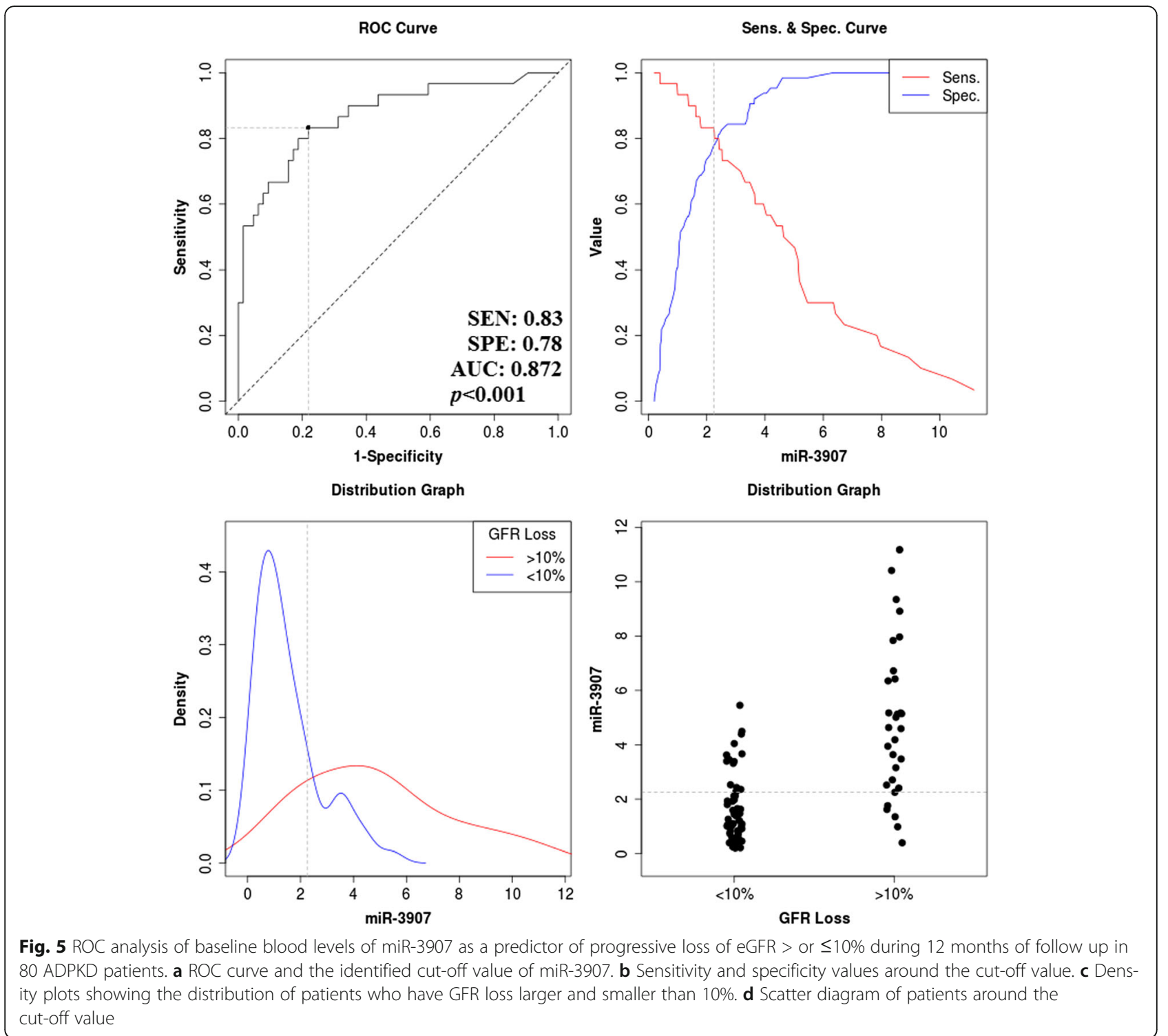

formation of the microdomains necessary for IP(3) $\mathrm{R} \mathrm{Ca}^{2}$ ${ }^{+}$-amplified $\mathrm{Ca}^{2+}$ release. Similarly, IP(3)RIP has been demonstrated to robustly enhance $\mathrm{Ca}^{2+}$-mediated inhibition of $\mathrm{IP}(3) \mathrm{R} \mathrm{Ca}(2+)$ release [16]. Speculatively an impaired PKD-function would thus lead to lower IP(3) $\mathrm{R} \mathrm{Ca}^{2}$ ${ }^{+}$-release, triggering a compensatory downregulation of IP(3)RIP through miR-targeting of its' mRNA.

The source cells and targets of the miRs identified in the present study may also the molecular pathogenesis of ADPKD. miR silencing of target mRNAs is mediated by complementary sequences (miR recognition element) located in the 3' untranslated region (UTR) of the mRNA matching approximately 2-8 nucleotides (the seed region) at the $5^{\prime}$ end of the miR. Thus, any given miR is likely to have more than one target mRNA. Despite this complexity, recent research has elucidated specific roles for individual miRs in kidney development and physiology [9, 10, 17]. In ADPKD rodent models miR-15a is down-regulated [8]. Moreover, the miR$17 \sim 92$ cluster is reported to be highly expressed in kidneys from several mouse models of PKD while its overexpression in normal mice produces renal cysts in wildtype mice $[8,18]$. Based on these data and increased urine levels of miR-13-3b, Ben-Dov et al. have proposed a role of miRs as biomarkers in ADPKD [5].

As shown in Fig. 1, the miRs with significant differences in circulating copy numbers between patients and controls in our study preferrentially involved certain pathways. Of these ErbB signaling is targeted by miR-126-5p and miR-27a-3p, and our data is in this regard consitent with earlier reports of ErbB as an important signal for cyst enlargement in ADPKD [19, 20]. 
Table 3 Univariate and multiple binary logistic regression models investigating the robustness of baseline blood miR-3907 as a predictor of GFR loss during 12 months of follow-up in 80 ADPKD patients. A decrease of $>10 \%$ of baseline eGFR at follow-up was defined as progression

\begin{tabular}{|c|c|c|c|c|c|c|}
\hline \multirow[t]{3}{*}{ Variable } & \multicolumn{2}{|l|}{ Univariate } & \multirow{2}{*}{\multicolumn{2}{|c|}{$\begin{array}{l}\text { Multivariate } \\
\text { Initial forward logistic regression model }\end{array}$}} & \multirow{2}{*}{\multicolumn{2}{|c|}{ Final logistic regression model }} \\
\hline & \multirow{2}{*}{$\begin{array}{l}\text { Progression OR } \\
(95 \% \mathrm{Cl})\end{array}$} & \multirow[t]{2}{*}{$p$} & & & & \\
\hline & & & Progression OR(95\% Cl) & $p$ & Progression OR(95\% CI) & $p$ \\
\hline Age (above vs. below median) & $1.11(1.05-1.18)$ & $<0.001$ & $1.30(1.07-1.58)$ & 0.008 & $1.28(1.09-1.51)$ & 0.003 \\
\hline Gender (if female) & $1.47(0.62-3.51)$ & & 3.59(0.62-20.89) & & & \\
\hline Hypertension (if present) & $9.58(2.64-34.79)$ & $<0.001$ & 0.75(0.03-19.49) & ns & & \\
\hline \multicolumn{7}{|l|}{ CKD stage } \\
\hline 4 & 1.00 & $<0.001$ & 1.00 & 0.032 & 1.00 & 0.014 \\
\hline 3 (vs. stage 4) & $1.35(0.26-7.07)$ & ns & $0.02(0.01-1.32)$ & ns & $0.02(0.01-0.70)$ & 0.032 \\
\hline 2 (vs. stage 4) & $0.08(0.01-0.49)$ & 0.007 & $0.01(0.01-0.12)$ & 0.007 & $0.01(0.01-0.07)$ & 0.004 \\
\hline 1 (vs. stage 4) & $0.08(0.01-0.49)$ & 0.006 & $0.01(0.01-0.62)$ & 0.027 & $0.01(0.01-0.37)$ & 0.011 \\
\hline miR-3907 (above vs. below median) & $2.37(1.66-3.40)$ & $<0.001$ & $3.21(1.63-6.32)$ & 0.001 & $3.06(1.65-5.68)$ & $<0.001$ \\
\hline
\end{tabular}

Bold values indicate the significant values $(p<0.05)$

Overexpression of ErbB4 in mice induced renal cysts [21], whereas deletion attenuated disease progression in the Cpk mouse model [22]. Finally Aguado-Fraile et al. have suggested that miR-27a-3p may be useful as a marker of acute kidney injury even in the absence of ADPKD [23].

Other signalling pathways regulated by the miRs significantly different between the groups in our data include apoptosis, where miR-92a-3p and miR-202-5p have both been reported to display phenotype-relavant protective roles in models of IgA [6]. Finally, miR-4516 isolated from urinary exosomes has been proposed as a urine biomarker of sodium re-uptake in the kidney [24]. Taken together, these findings suggest that the miRs identified in our data are not random noise but reflect an underlying biology of a significance yet to be determined.

A number of weaknesses in the present study must be acknowledged. The absence of a non-ADPKD CKD control group is a weakness, especially as our data suggests that eGFR is a significant determinant of several miRs. Also, the limited number of patients combined with the lack of ultrasonographically measured renal volumes also limits the interpretation of the data. Nonetheless our data are among the first measuring miRs in ADPKD and may serve as a foundation for future candidate-based studies.

\section{Conclusions}

The present study suggests (a) that random bloodsampling in a clinical setting can yield meaningful patterns of miRs, (b) that these patterns vary between healthy subjects and those with kidney disease, and, finally, (c) that increased copy numbers of miR-3907 in the circulation predicts the future decline in eGFR among ADPKD patients followed for 12 months.

\section{Additional file}

Additional file 1: List of miRNAs included in the Qiagen miScript miRNA PCR Array Human Serum \& Plasma 384HC in Excel format. (XLSX 12.5 KB)

\section{Abbreviations}

ADPKD: Adult polycystic kidney disease; ESRD: Eend-stage renal disease; eGFR: Estimated glomerular filtration rate from biochemical data; miRs: micro-RNAs; qPCR: Reverse transcription reaction and quantitative real time polymerase chain reaction; ROC: Reciever operating characteristics methodology

\section{Acknowledgements}

We gratefully acknowledge the practical support provided by the clinical staff at the participating hospitals as well as the administrative support of the Turkish Society of Nephrology.

\section{Funding}

This study was supported by Research Foundation of the Erciyes University (Project Number:TSA-2013-4583), the Marianne and Marcus Wallenbergs stiftelse $(J A)$, the Swedish Heart and Lung Foundation (JA) and the Swedish Medical Research Council (JA). The supporting organizations had no influence on the design, implementation or distribution of the study and its' results, nor did they participate in data analyses or have access to manuscripts before publication.

\section{Availability of data and materials}

All original data (including electronic copies of imaging studies) are available for review at the Department of Nephrology of Erciyes University, Kayseri, Turkey. Access is conditional only on signed consent to respect the privacy of the individual patients involved (University mandated declaration form to be signed).

\section{Authors' contributions}

IK, EE, FO, AU, GZ, MHS, BT, OO participated in the design of the study, patient recruitment and follow-up, data entry and analysis, and also in interpretation of data and writing of this manuscript. ST and EFS participated in the study design, performed all of the imaging studies and measurements, as well as assisted with data interpretation and reviewed the final manuscript. KK participated in the study design, performed the miR analyses and helped interpret the result, as well as reviewed the final manuscript. GZ and YO participated in the study design, performed all statistical analyses and helped interpret the result, as well as reviewed the final manuscript. TE and JA came up with the original research question, participated in the study design, helped interpret the results, as well 
as wrote the final manuscript. All authors have read and approved the current version of the manuscript.

\section{Competing interests}

The authors declare that they have no competing interests.

\section{Consent for publication.}

Not applicable.

\section{Ethics approval and consent to participate}

The study protocol was approved by the Erciyes University Ethical Review Board (Kayseri, Turkey) prior to start, and all patients gave signed informed consent prior to inclusion.

\section{Publisher's Note}

Springer Nature remains neutral with regard to jurisdictional claims in published maps and institutional affiliations.

\section{Author details}

${ }^{1}$ Department of Internal Medicine, Division of Nephrology, Erciyes University Medical Faculty, Kayseri, Turkey. ${ }^{2}$ Department of Medical Biology, Erciyes University Medical Faculty, Kayseri, Turkey. ${ }^{3}$ Department of Internal Medicine, Erciyes University Medical Faculty, Kayseri, Turkey. ${ }^{4}$ Betul-Ziya Eren Genome and Stem Cell Center, Erciyes University Medical Faculty, Kayseri, Turkey. ${ }^{5}$ Department of Biostatistics, Erciyes University, Kayseri, Turkey. ${ }^{6}$ Department of Internal Medicine, Division of Nephrology, Istanbul Bilim University, Istanbul, Turkey. ${ }^{7}$ Division of Vascular Biology, Department of Medical Biochemistry and Biophysics, Karolinska institutet, Karolinska University Hospital, Stockholm, Sweden. ${ }^{8}$ Department of Clinical Immunology, Karolinska University Hospital, C2:66 ImmTrans, 14186 Stockholm, Sweden.

Received: 1 December 2016 Accepted: 23 May 2017

\section{Published online: 30 May 2017}

\section{References}

1. Torres VE, Harris PC, Pirson Y. Autosomal dominant polycystic kidney disease. Lancet. 2007:369(9569):1287-301.

2. Schrier RW, et al. Predictors of Autosomal Dominant Polycystic Kidney Disease Progression. J Am Soc Nephrol. 2014;25(11):2399-418.

3. Grantham JJ, et al. Volume Progression in Polycystic Kidney Disease. N Engl J Med. 2006;354(20):2122-30.

4. Wing MR, et al. Epigenetics of Progression of Chronic Kidney Disease: Fact or Fantasy? Semin Nephrol. 2013;33(4):363-74.

5. Ben-Dov IZ, et al. Urine MicroRNA as Potential Biomarkers of Autosomal Dominant Polycystic Kidney Disease Progression: Description of miRNA Profiles at Baseline. PLoS One. 2014;9(1):e86856.

6. Duan Z-Y, et al. Selection of urinary sediment miRNAs as specific biomarkers of IgA nephropathy. Sci Rep. 2016;6:23498.

7. Liep J, et al. Cooperative Effect of miR-141-3p and miR-145-5p in the Regulation of Targets in Clear Cell Renal Cell Carcinoma. PLoS One. 2016;11(6):e0157801

8. Patel V, et al. miR-17 92 miRNA cluster promotes kidney cyst growth in polycystic kidney disease. Proc Natl Acad Sci. 2013;110(26):10765-70.

9. Pandey $P$, et al. Microarray-based approach identifies microRNAs and their target functional patterns in polycystic kidney disease. BMC Genomics. 2008;9(1):624.

10. Pandey $P$, et al. Systems biology approach to identify transcriptome reprogramming and candidate microRNA targets during the progression of polycystic kidney disease. BMC Syst Biol. 2011;5(1):56.

11. Smyth GK. An efficient algorithm for REML in heteroscedastic regression. J Comput Graph Stat. 2002;11(4):836-47.

12. Vlachos IS, et al. DIANA-miRPath v3.0: deciphering microRNA function with experimental support. Nucleic Acids Res. 2015;43(W1):W460-6.

13. Kocyigit I, et al. Early Arterial Stiffness and Inflammatory Bio-Markers in Normotensive Polycystic Kidney Disease Patients. Am J Nephrol. 2012;36(1):11-8.

14. Agarwal $\mathrm{V}$, et al. Predicting effective microRNA target sites in mammalian mRNAs. elife. 2015;4

15. Sammels $E$, et al. Polycystin-2 activation by inositol 1,4,5-trisphosphate-induced Ca2+release requires its direct association with the inositol 1,4,5-trisphosphate receptor in a signaling microdomain. J Biol Chem. 2010;285(24) doi:10.1074/ jbc.M109.090662
16. Devogelaere $B$, et al. The complex regulatory function of the ligandbinding domain of the inositol 1,4,5-trisphosphate receptor. Cell Calcium. 2008;43(1):17-27.

17. Lee S-O, et al. MicroRNA15a modulates expression of the cell-cycle regulator $\mathrm{Cdc} 25 \mathrm{~A}$ and affects hepatic cystogenesis in a rat model of polycystic kidney disease. J Clin Investig. 2008;118(11):3714-24.

18. Sun $\mathrm{H}$, et al. MicroRNA-17 post-transcriptionally regulates polycystic kidney disease-2 gene and promotes cell proliferation. Mol Biol Rep. 2009;37(6):2951-8.

19. Harskamp $L R$, et al. The epidermal growth factor receptor pathway in chronic kidney diseases. Nat Rev Nephrol. 2016;12(8):496-506.

20. Stocklin E. An activated allele of the c-erbB-2 oncogene impairs kidney and lung function and causes early death of transgenic mice. J Cell Biol. 1993; 122(1):199-208.

21. Veikkolainen V, et al. ErbB4 Modulates Tubular Cell Polarity and Lumen Diameter during Kidney Development. J Am Soc Nephrol. 2011;23(1):112-22.

22. Zeng F, et al. Deletion of ErbB4 accelerates polycystic kidney disease progression in cpk mice. Kidney Int. 2014;86(3):538-47.

23. Aguado-Fraile E, et al. A Pilot Study Identifying a Set of microRNAs As Precise Diagnostic Biomarkers of Acute Kidney Injury. PLoS One. 2015;10(6):e0127175.

24. Salih $M$, et al. Urinary extracellular vesicles as markers to assess kidney sodium transport. Curr Opin Nephrol Hypertens. 2016;25(2):67-72.

\section{Submit your next manuscript to BioMed Central and we will help you at every step:}

- We accept pre-submission inquiries

- Our selector tool helps you to find the most relevant journal

- We provide round the clock customer support

- Convenient online submission

- Thorough peer review

- Inclusion in PubMed and all major indexing services

- Maximum visibility for your research

Submit your manuscript at www.biomedcentral.com/submit
) Biomed Central 